\title{
Towards a Practical, Non-enzymatic Process for Molnupiravir from Cytidine
}

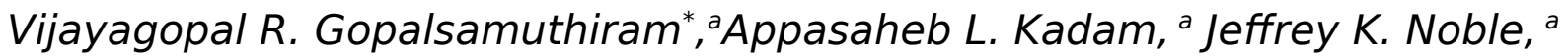 \\ David R. Snead, a Corshai T. Williams, ${ }^{b}$ Timothy F. Jamison, ${ }^{b}$ Chris Senanayake, ${ }^{c}$ \\ Ajay Yadaw, ' Sarabindu Roy, 'Gopal Sirasani, ' Frank B. Gupton, a Justina M.

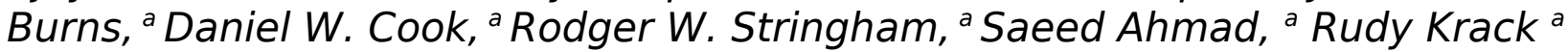

${ }^{a}$ Medicines for All Institute, 737 N 5th St., Box 980100, Richmond, Virginia 23298, ${ }^{\text {b }}$ Department of Chemistry, Massachussetts Institute of Technology, 77 Massachusetts Ave, Cambridge, MA 02139-4307, 'TCG Green Chem, Process R \& D center, Princeton south, Ewing, NJ 08628

KEYWORDS: COVID-19, antivirals, molnupiravir, EIDD-2801, MK-4482

ABSTRACT: A scalable four step synthesis of molnupiravir from cytidine is described herein. The attractiveness of this approach is its fully chemical nature involving inexpensive reagents and more environmentally friendly solvents such as water, isopropanol, acetonitrile and acetone. Isolation and purification procedures are improved in comparison to our earlier report, as all intermediates can be isolated via aqueous acid treatment and recrystallization. The key steps in the synthesis, namely ester formation, hydroxamination and deprotection were done on multigram scale to afford molnupiravir in 36-41\% yield with average purity of 98 wt $\%$ by q-NMR and 99 area \% by HPLC.

\section{Introduction}

Molnupiravir (also known as EIDD-2801 and MK-4482) is a promising drug candidate for treating COVID-19. Merck licensed the compound from Ridgeback Biotherapeutics in 2020, and clinical results with move-out patients advanced molnupiravir to Phase 3 clinical trials. ${ }^{1}$ Molnupiravir offers complementary advantages to remdesivir, such as oral bioavailablity and structural simplicity, thus reducing the manufacturing complexity. The original synthesis of molnupiravir developed by Painter utilizes uridine $\mathbf{1}$ and proceeds in five steps in low overall yield (17\% overall, two steps have assumed yields) with the aid of an acetonide protecting group strategy (Scheme 1$){ }^{5}$

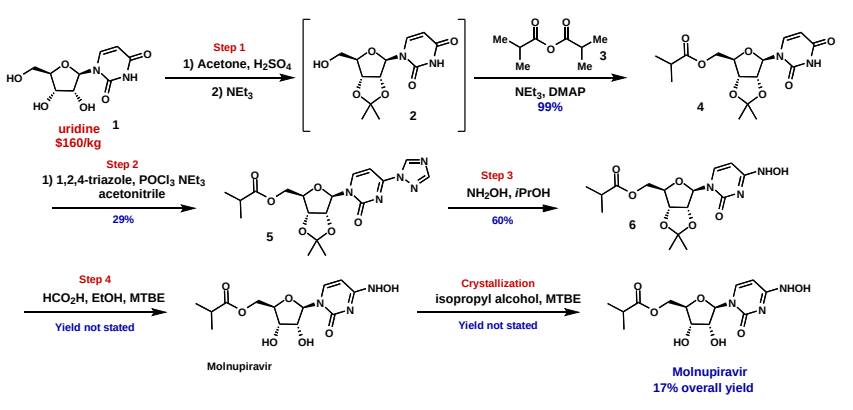

Scheme 1. Discovery route to Molnupiravir

As the above synthesis had high step count accompanied by a low yield, an alternative synthesis of molnupiravir was necessary. In our original synthetic route, we found that Novozyme 435, a common lipase enzyme, could selectively esterify the primary hydroxy groups of cytosine derivatives without the need for acetonide protection, thus reducing the overall step count to two (Route I, Scheme 2). ${ }^{6,9}$ The order of this two-step sequence can be varied either by doing the hydroxamination of cytidine (7) first followed by regioselective acylation or vice-versa. In a recent preprint disclosure, Merck demonstrated a similar enzymatic strategy starting from ribose $\mathbf{9}$ and cytosine $\mathbf{1 4}$ proceeding in 69 $\%$ yield over three steps. ${ }^{7}$ Although the enzymatic routes are indeed attractive because of the low step count, we felt that demonstrating a scalable, non-enzymatic reaction sequence would still be valuable to ensure maximum global access to this important drug candidate. Our preliminary results on a fully chemical route to molnupiravir (Route II, Scheme 2) were disclosed recently. ${ }^{6 b}$ 


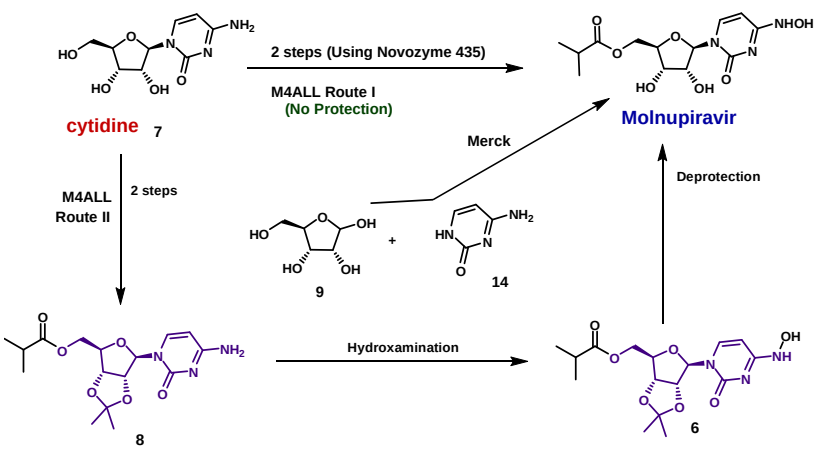

Scheme 2. Routes to Molnupiravir

The details of our acetonide approach to molnupiravir are shown in Scheme 3. As with our other routes, this chemistry began with protection of $\mathbf{7}$ as its acetonide $\mathbf{1 0}$ in $94 \%$ yield using 2,2dimethoxypropane in conjunction with acetone and sulfuric acid. Chemo-selective esterification was then accomplished using isobutyric anhydride and DBU in acetonitrile to afford the ester $\mathbf{8}$ in $78 \%$ isolated yield. Hydroxamination gave the penultimate intermediate 6 in excellent yield $(96 \%)$, and upon deprotection with formic acid, molnupiravir was isolated in $42 \%$ overall yield for the four steps. Alternatively, we can reduce one step in this sequence by using a freshly prepared solution of 60/40 water/IPA, whereby molnupiravir is obtained in a single step from $\mathbf{8}$ in an overall 39\% yield (Step 3'). While this three-step approach appears attractive, challenges foreseen to control the impurity profile of molnupiravir due to telescoping the entire operation made it less favorable for further development. The products from Step 1 and 3 were isolated by crystallization; however, column chromatography was used to isolate the products step 2 and molnupiravir. ${ }^{6 \mathrm{~b}}$ This prompted us to explore further optimization to avoid some of these drawbacks.
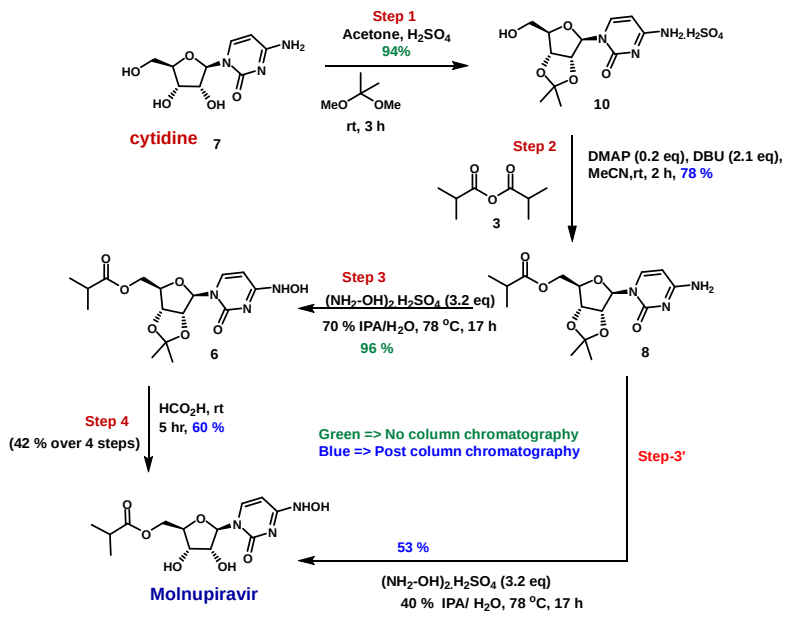

Scheme 3. Acetonide Approach to molnupiravir

As a follow up to this earlier work, we now report herein an optimized, operationally simpler process complementary to the enzymatic approach featuring a chromatography-free isolation of intermediates from Step 2 to Step 4. The scalability of the entire process has been demonstrated by synthesizing multigram quantities of molnupiravir.

\section{Results and discussion}

\section{- Impurity profiling in Steps 2, 3 and 4}

Prior to further developmental work, we identified the impurities in each step of our route either by direct isolation from the reaction mixtures or by synthesis of authentic materials for comparison with reaction in-process control data (Figure 1). The key impurities present in Step 2 were found to be the diacylated side product 11 and the $N$-acylated side product 12. For Step 3, the key impurities present in the crude reaction mixture were the starting amine 8, molnupiravir, 13, unreacted hydroxylamine sulfate besides the hydroxaminated product $\mathbf{6}$. For Step 4, the key impurities were 6, 8, EIDD-1931, 13 and uridine 1.

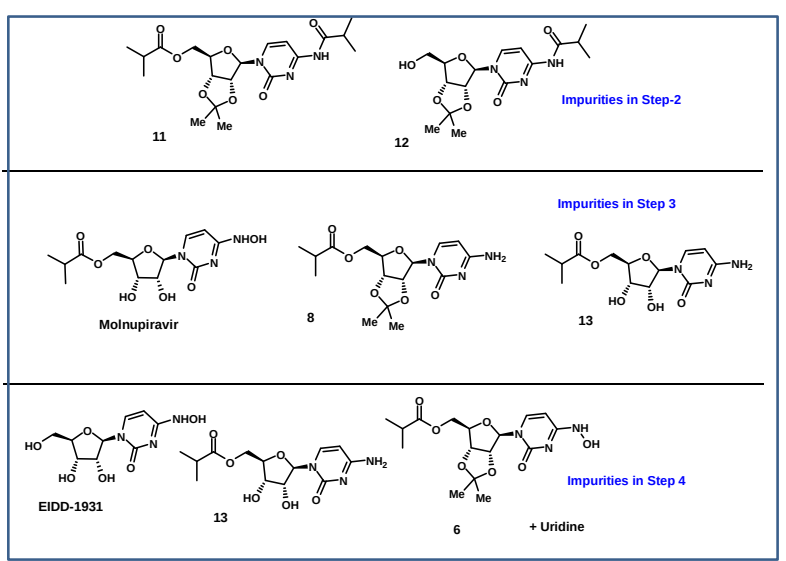

Figure 1. Side products in Step 2, Step 3 and Step 4

\section{- Step 2 Esterification: Impurity Profile and Reaction Optimization}

A time profile for the acylation of $\mathbf{1 0}$ was conducted to follow the formation of product and impurities in this reaction (See Figure S1, Supporting information). Within 30 minutes, the reaction proceeds to more than $85 \%$ conversion, but conversion quickly tapers off. Over an additional 17-hour reaction period, only a marginal increase in product formation is observed and no additional starting material is consumed. Over this same time period, an over-acylated side product $\mathbf{1 1}$ grows in after a short lag behind the product, ultimately reaching a total of 6.25 area\%, and the undesired $\mathrm{N}$-acylated side product $\mathbf{1 2}$ is also observed, albeit in very small amounts $(0.2$ area\%). 
Although side product $\mathbf{1 1}$ constitutes a non-negligible portion of the final reaction mixture, we were curious to know if this material could be transformed to the desired product of the subsequent step, the transamination with hydroxylamine. If possible, this may eliminate the need for purging this impurity while providing a yield boost for the subsequent step. To probe this possibility, authentic $\mathbf{1 1}$ was prepared from the $O$ monoacylated 8 by reaction iso-butyryl chloride. Once isolated, 11 was treated with 3.2 equivalents of hydroxylamine sulfate. This reaction led to the formation of at least seven compounds by HPLC, of which the major constituents were 9 and molnupiravir in 21 and 31 area\%, respectively. Although we were disappointed that this reaction did not lead to the desired compound $\mathbf{6}$, it did provide some useful insights for Step 3. Specifically, this reaction profile matches that seen for the hydroxamination step (Step 3), which suggests that the source of impurities in that reaction stems from side reactions arising from 11 (Scheme 4). This experiment clearly demonstrates that minimization of side product 11 in Step 2 is critical.

A systematic optimization of key variables in Step 2 was conducted in ordered to maximize the formation of the monoacylated product $\mathbf{8}$ and minimize the diacylation side reaction leading to compound 11. The results of this optimization screen are shown in Table 1. The most impactful result from this parameter screen involves the reaction concentration. Because the formation of $\mathbf{1 1}$ requires two consecutive second-order reactions, we reasoned that decreasing the overall reaction concentration would lead to a dramatic decrease in the rate of the second acylation reaction. Indeed, increasing the amount of solvent from 2.5 to $10 \mathrm{~V}$ (Table 1, entries 5-7) increased the area\% of 8 from 89 to $94 \%$ and reduced the overall contribution from the impurities 10, $\mathbf{1 1}$ and $\mathbf{1 2}$ from $11 \%$ to $4.3 \%$ (Table 1, Figure S2 Supporting information).

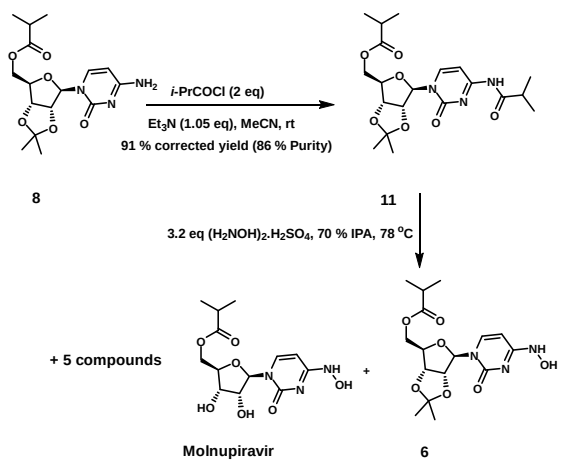

Scheme 4. Synthesis of diacylated side product 11 and its subsequent hydroxamination products

Table 1. Impact of concentration, temperature and base on product distribution (HPLC at $260 \mathrm{~nm}$ )

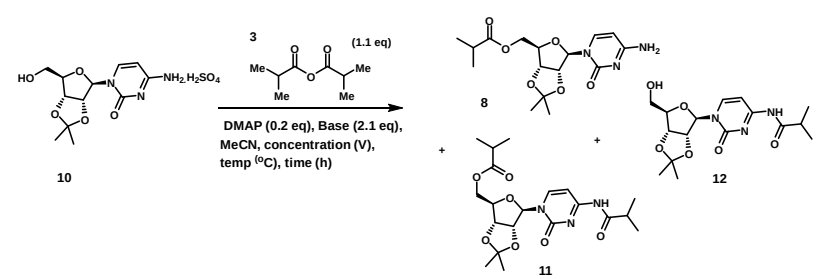

\begin{tabular}{|l|l|l|l|l|l|l|l|}
\hline Entry & Base & Temp & $\begin{array}{l}\text { Solv } \\
\text { Vol }\end{array}$ & $\begin{array}{l}\text { Area } \\
\%\end{array}$ & $\begin{array}{l}\text { Area } \\
\% \\
10\end{array}$ & $\begin{array}{l}\text { Area } \\
\% \\
11\end{array}$ & $\begin{array}{l}\text { Area } \\
\% \\
12\end{array}$ \\
\hline 1 & $\mathrm{Et}_{3} \mathrm{~N}$ & 19 & 10 & 59 & 0 & 17 & 0.5 \\
\hline 2 & $\mathrm{Bu}_{3} \mathrm{~N}$ & 19 & 10 & 52.6 & 0 & 21.6 & 1.1 \\
\hline 3 & DIPEA & 19 & 10 & 60.4 & 0 & 17.5 & 0 \\
\hline 4 & Lutidine & 19 & 10 & 34.5 & 0 & 30.2 & 6.5 \\
\hline 5 & DBU & 19 & 10 & 94.7 & 2.2 & 2.1 & 0.1 \\
\hline 6 & DBU & 19 & 2.5 & 89.3 & 7.1 & 3.6 & 0.3 \\
\hline 7 & DBU & 19 & 5 & 89.4 & 5.35 & 4.3 & 0.3 \\
\hline 8 & DBU & 19 & 7 & 92.5 & 0.4 & 6.1 & 0.1 \\
\hline 9 & DBU & 0 & 10 & 94.7 & 2.25 & 2.1 & 0.6 \\
\hline 10 & DBU & 40 & 10 & 88.7 & 6 & 4.6 & 0.4 \\
\hline 11 & DBU & 56 & 10 & 85.5 & 8.5 & 5.4 & 0.6 \\
\hline
\end{tabular}

A screen of various bases confirmed that DBU is best choice for maximum conversion to the mono-acylated product $\mathbf{8}$ (Table 1, entries 1-5).

Lastly, a screening of the reaction temperatures using DBU as the base at a reactant concentration of 10 volumes led to identification of $0{ }^{\circ} \mathrm{C}$ as the optimal reaction temperature affording maximum product formation (Table 1, entries 5 vs 911).

Although DBU was found to be the optimal base for this reaction, its removal from the reaction mixture is challenging. In our first report, chromatographic separation was used to isolate the pure compound, but this will be costly on scale. Given the large $\mathrm{pK}_{\mathrm{a}}$ difference between DBU $(\sim 12.5)$ and the cytidine amine functionality $(\sim 4.2)$, we reasoned that a mild acidic workup could potentially remove DBU from the organic layer without extraction of the product $\mathbf{8}$. Several aqueous acid washes were screened (10\% ammonium chloride, $10 \%$ acetic acid, $10 \%$ sulfuric acid and neat $\mathrm{H}_{3} \mathrm{PO}_{4}$ ), and $10 \%$ acetic acid resulted in the maximum recovery of the ester post workup with saturated sodium bicarbonate solution. The purity of the final product was 
found to be $84 \mathrm{wt} \%$ by qNMR in chloroform-d, and by HPLC at $260 \mathrm{~nm}$, this corresponded to 92 area $\%$ of $\mathbf{8}, 4.7$ area\% of $\mathbf{1 1}$ and 0.44 area $\%$ of $\mathbf{1 2}$.

The scalability and reproducibility of these optimized reaction conditions were demonstrated in two reproduction runs as shown in Table 2. The corrected yields and product purities were found to be comparable across the different scales of the reactions. A comparison of HPLC data for an in-process control vs the organic layer post $10 \%$ acetic acid and bicarbonate workup shows that the relative ratios of cytosine-containing materials were relatively unaffected by the extraction conditions. Unfortunately, a trace amount of DBU was detected $(0.47$ area \%) in the final product. (Figure S3, Supporting information).
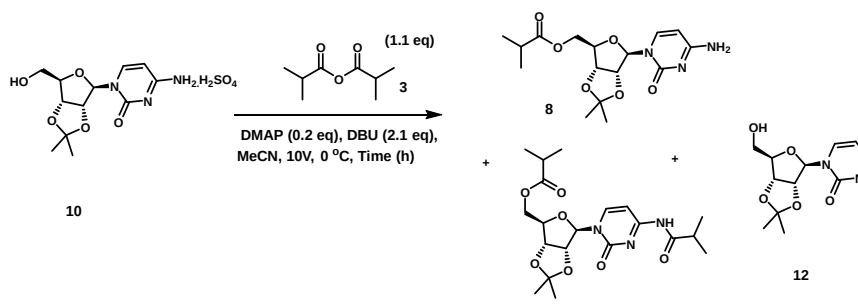

Table 2: Scale up of the low temperature esterification (HPLC at 260 $\mathrm{nm})$

\begin{tabular}{|c|c|c|c|c|c|c|c|c|}
\hline $\begin{array}{l}\text { Entr } \\
\text { y }\end{array}$ & $\begin{array}{l}\text { Scal } \\
\mathrm{e}\end{array}$ & $\begin{array}{l}\text { Tim } \\
\mathrm{e}\end{array}$ & $\begin{array}{l}\text { Purit } \\
y(\%) \\
\text { of } 10\end{array}$ & $\begin{array}{l}\% \\
\text { Adjuste } \\
\text { d Yield } \\
\text { of } 8\end{array}$ & $\begin{array}{l}\% \\
\text { Purit } \\
y \text { of } \\
8\end{array}$ & $\begin{array}{l}\text { \% Side } \\
\text { produ } \\
\text { ct } 11\end{array}$ & $\begin{array}{l}\text { Area } \\
\% 12^{\mathrm{a}}\end{array}$ & $\begin{array}{l}\% \\
\text { Purit } \\
y \text { of } \\
11\end{array}$ \\
\hline 1 & $\begin{array}{l}9.6 \\
\mathrm{~g}\end{array}$ & $1 \mathrm{~h}$ & $96 \%$ & $89 \%$ & $85 \%$ & $4.1 \%$ & $0.3 \%$ & $\begin{array}{l}4.8 \\
\%\end{array}$ \\
\hline 2 & $20 \mathrm{~g}$ & $\begin{array}{l}1.5 \\
\mathrm{~h}\end{array}$ & $91 \%$ & $81.8 \%$ & $88 \%$ & $4.2 \%$ & & $\begin{array}{l}4.8 \\
\%\end{array}$ \\
\hline
\end{tabular}

- Step 3 Hydroxamination: Impurity profile and reaction optimization

In our preliminary report, the transamination of $\mathbf{8}$ using hydroxylamine sulfate resulted in $96 \%$ yield ( $94 \mathrm{wt} \%$ purity) of 9. Unreacted hydroxylamine sulfate was removed by filtration after dissolution of $\mathbf{9}$ in a minimal amount of acetonitrile. However, in our previous report, the starting material for this reaction had been purified by column chromatography. Conducting the same transamination reaction with ester 8 (84 wt $\%$ purity) that was obtained using our optimized acetic acid workup gives a mixture of the product $\mathbf{6}$, molnupiravir and some unreacted starting material (Scheme 5). A typical distribution of products at the end of $20 \mathrm{~h}$ reaction period with an internal reaction temperature of $73{ }^{\circ} \mathrm{C}$ is 86 area\% product 6,6 area\% of starting material 8, 3 area $\%$ molnupiravir and $\sim 1$ area $\%$ of the acetonide-deprotected ester 13. After isolation, this translated to $89 \%$ average adjusted isolated yield with $73 \mathrm{wt} \%$ purity from duplicate runs before removal of unreacted hydroxylamine sulfate.

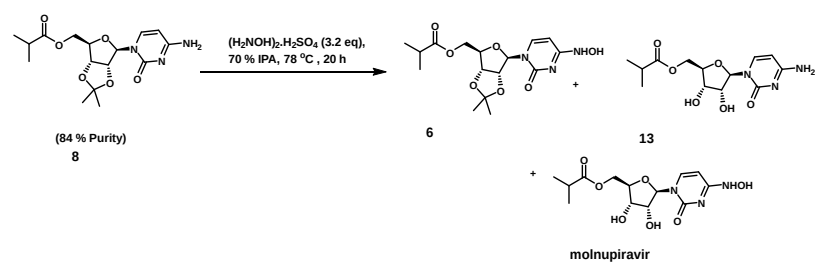

Scheme 5. Hydoxamination and side products formed

For purification of the hydroxaminated product $\mathbf{6}$, two distinct approaches were identified. The first method (Method A) involves recrystallization of crude 6 (73 wt $\%$ purity) from $2.5 \mathrm{~V}$ of isopropyl acetate. The mass recovery using this method is $66 \%$ and the purity is $98 \mathrm{wt} \%$ by qNMR in acetone- $d_{6}$ (See Figure S4, Table S1, supporting information). In the second purification method (Method B), recrystallization of the crude product from a minimal amount of acetonitrile provides $64 \%$ adjusted isolated yield of 6 with a purity of $96 \mathrm{wt} \%$ by q-NMR. While more 6 was also present in the mother liquor from Method A, additional product from Method B was isolated by concentration of the mother liquor recrystallizing the remaining solid in $2.5 \mathrm{~V}$ of isopropyl acetate. This secondary isolation affords an additional $11 \%$ mass yield of material with purity of $90 \mathrm{wt} \%$ by qNMR(Scheme 6).

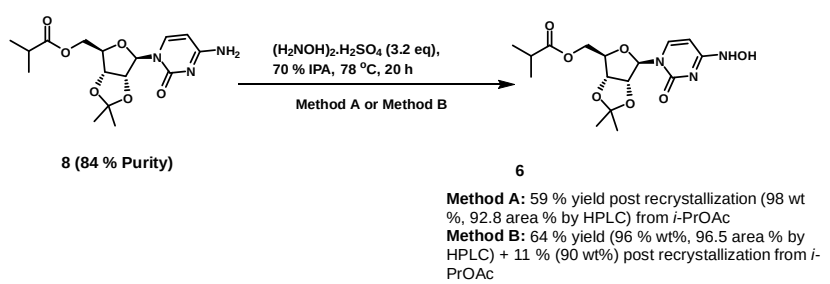

Scheme 6. Isolated yields post purification via Method A or Method B

The two methods described above (with recrystallization or by trituration) afford Step 3 product 6 of > $96 \%$ purity. More notably, the amount of $\mathbf{1 3}$ was reduced post purification by either of these methods from 1.7 area\% to 0.11 area\% (See HPLC trace in Figure S6, peak at retention time $4.998 \mathrm{~min}$ ). The key impurities in 6 obtained by either of these methods were molnupiravir and 8 (2.6 area \% and 0.27 area \% by Method A, 2.1 area $\%$ and 0.52 area \% by Method B). Method A was preferred for scale up as it provided 6 of higher purity. 
Although the product of this reaction is contaminated with a small amount of $\mathbf{1 3}$, this material is known to react with hydroxylamine to form molnupiravir.To verify this assertion, an independently prepared sample of $\mathbf{1 3}$ was converted to molnupiravir in $\mathbf{7 8 \%}$ assay yield of crude material (40 wt $\%$ purity, Scheme 7 ). ${ }^{6}$

\section{- Step 4 Deprotection: Impurity profile and optimization}

With access to relatively pure 6 from Step 3, screening of conditions for deprotection of the acetonide group in $\mathbf{6}$ was next performed (See Scheme 8 and Figure 2). Most protic acids such as sulfuric acid, phosphoric acid, hydrochloric acid, or Lewis acids, such as zirconium tetrachloride, provided molnupiravir in low to modest yields $(<50 \%)$ (as estimated by HPLC area\% of the crude reaction mixtures), along with a variety of side products.

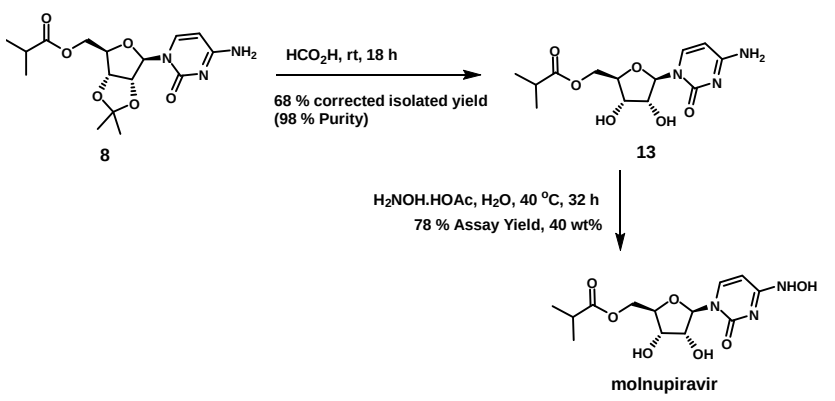

Scheme 7. Acetonide deprotection of 8 and the subsequent conversion

of monoester 13 to molnupiravir

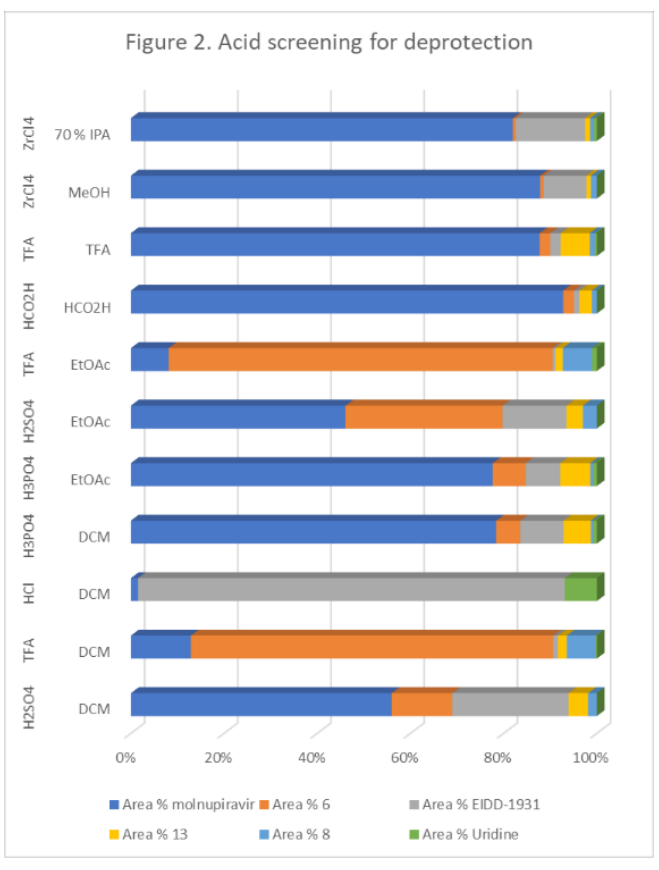

For example, increased formation of $\mathbf{8}$ and $\mathbf{1 3}$ under some reaction conditions (TFA in EtOAc, TFA in DCM or $\mathrm{HCl}$ in DCM, $\mathrm{H}_{2} \mathrm{SO}_{4}$ in DCM) possibly indicates a self-oxidation reduction, previously reported with aryl hydroxylamines. ${ }^{8}$ Of all the acids screened, only neat formic acid or trifluoroacetic acid provide conversions to molnupiravir $>90 \%$.

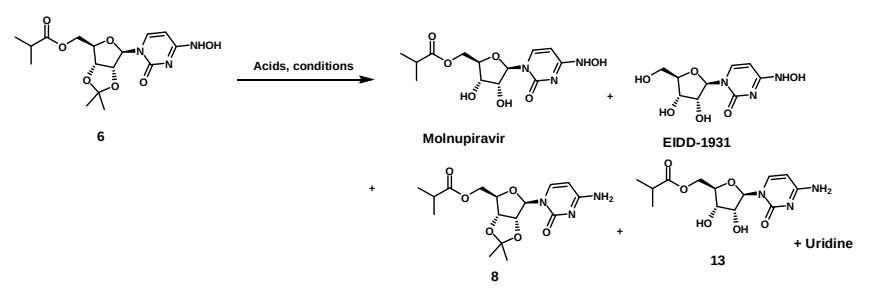

Scheme 8. Acid screening for deprotection to molnupiravir

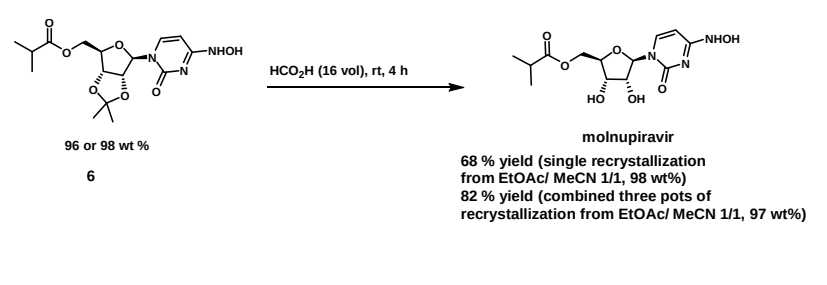

Scheme 9. Step 4 yields post recrystallization

A wide variety of recrystallization conditions were screened to improve the purity of molnupiravir and the results are as shown in Table S2, Supporting information. Using the optimal conditions 1:1 ethyl acetate/ acetonitrile, the deprotection of the acetonide was scaled up to $26 \mathrm{~g}$ affording an $82 \%$ combined yield of the recrystallized material from three crops in successive recrystallizations of the residue from the mother liquor with purity at $97 \mathrm{wt} \%$ by qNMR in methanol- $d_{4}$ (Scheme 9 , Figure S8 Supporting Information). However, either water or a combination of $n$-butanol with water was preferred for recrystallization as it afforded molnupiravir with much higher purity (See Table S3, entries $3 \& 4$, Supporting information) but with a lower mass recovery.

\section{- Scalability}

A preliminary evaluation of the scalability of the entire sequence was conducted at $100 \mathrm{~g}$ scale. Acetonide protection proceeded to afford the product of Step 1 in excellent yield and purity (Table $3)$. On a $150 \mathrm{~g}$ scale, the optimized Step 2 conditions resulted in $>90 \%$ isolated yield with good purity; however, there was some discrepancy in the purity of the isolated material between the two batches. At $130 \mathrm{~g}$ scale, the hydroxamination (Step 3) showed only modest yields of product (70-76\%) post recrystallization with isopropyl acetate. The final deprotection affords 
molnupiravir in 59-61\% yield, which represents an overall yield of $36-41 \%$ over the 4 steps. The filtrate from the final recyrstallization contained additional molnupiravir (14\%). The impurity profile of the molnupiravir obtained from this route (Figure 10, Supporting information) indicates the presence of 6 $(0.31$ area \%) and $8(0.81$ area \%) as the two main impurities (Figure S9, Supporting information).

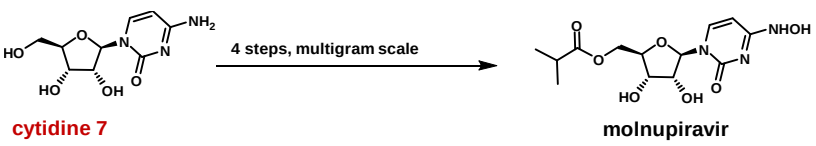

Table 3. Scale up of the 4-step sequence to molnupiravir

\begin{tabular}{|l|l|c|c|c|}
\hline Step & Scale & $\begin{array}{l}\% \text { Corrected } \\
\text { Isolated Yield }\end{array}$ & $\begin{array}{l}\text { Area \% HPLC } \\
(260 \mathrm{~nm})\end{array}$ & $\begin{array}{l}\text { Wt \% by } \\
\text { NMR }\end{array}$ \\
\hline \multirow{2}{*}{1} & $100 \mathrm{~g}$ & $94 \%$ & $99.1 \%$ & $95 \%$ \\
\cline { 2 - 5 } & $300 \mathrm{~g}$ & $92 \%$ & $99.2 \%$ & $95.5 \%$ \\
\hline \multirow{2}{*}{2} & $150 \mathrm{~g}$ & $92 \%$ & $98.6 \%$ & $89 \%$ \\
\cline { 2 - 5 } & $140 \mathrm{~g}$ & $95.2 \%$ & $94 \%$ & $85.8 \%$ \\
\hline \multirow{2}{*}{3} & $130 \mathrm{~g}$ & $70 \%$ & $97.3 \%$ & $94 \%$ \\
\cline { 2 - 5 } & $120 \mathrm{~g}$ & $76 \%$ & $98.7 \%$ & $95 \%$ \\
\hline 4 & $80 \mathrm{~g}$ & $59 \%$ & $98.6 \%$ & $98.5 \%$ \\
\hline \multirow{2}{*}{} & $100 \mathrm{~g}$ & $61 \%$ & $99.6 \%$ & $97.6 \%$ \\
\hline
\end{tabular}

\section{Conclusions}

In summary, improvements and reaction details for our fourstep route to molnupiravir from highly available cytidine are disclosed. Compound $\mathbf{1 0}$ from Step 1 is isolated directly from the reaction by filtration and washing whereas 8 from Step 2 is obtained directly by washing the organic layer with $10 \%$ acetic acid. Pure 9 from Step 3 is obtained by recrystallization of the crude from isopropyl acetate and molnupiravir from Step 4 is obtained by recrystallization from water. We have demonstrated comparable overall yields to our previous report and we have also been able to substitute column chromatographic purification with simple purification procedures that can be performed at scale.

\section{Acknowledgements}

This work was supported, in whole or in part, by the Bill \& Melinda Gates Foundation [Grant Number: OPP1176590]. Under the grant conditions of the Foundation, a Creative Commons Attribution 4.0 Generic License has already been assigned to the Author Accepted Manuscript version that might arise from this submission. We also thank Ryan Nelson for his inputs in editing the manuscript and Trevor Laird, John Dillon for reviewing this manuscript.

\section{Conflict of Interest}

The authors declare no competing financial interest.

\section{References}

1. a) Recent news articles see Kropff, A. "Coronavirus" https://www.wtsp.com/article/news/health/coronavirus/antiviraldrug-molnupiravir-showing-promise-in-trials-againstcoronavirus/67-87f24932-8244-439a-a664-ba6aa21aac01, Published Mar 26, 2021 b) Merck media "News release" https:// www.merck.com/news/merck-and-ridgeback-biotherapeuticsprovide-update-on-progress-of-clinical-development-programfor-molnupiravir-an-investigational-oral-therapeutic-for-the$\underline{\text { treatment-of-mild-to-moderate-covid-19/ }}$

2. Sheahan, T.P.; Sims, A.C.; Zhou, S.; Graham, R.L.; Pruijssers, A.J.; Agostini, M.L.; Leist, S.R.; Schafer, A.; Dinnon III, K.H.; Stevens, L.J.; Chappel, J.D.; Lu, X.; Hughes, T.M.; George, A.S.; Hill, C.S.; Montgomery, S.A.; Brown, A.J.; Bluemling, G.R.; Natchus, M.G.; Saindane, M.; Kolykhalov, A.A.; Painter, G.; Harcourt, J.; Tamin, A.; Thornburg, N.J.; Swanstrom, R.; Denison, M.R.; Baric, R.S. Sci. Transl. Med., 2020, 12, eabb5883.

3. Cox, R.M.; Wolf, J.D.; Plemper, R.K. Nat. Microbiol., 2021, 6, 11-18.

4. a) Halford, B. Chem. Eng. News 2020, 98, 22. b) Cross, R. Chem. Eng. News 2020, 98, 12.

5. a) Painter, G.R.; Bluemling, G.R.; Natchus, M.G.; Guthrie, D. WO2019113462, 2018. b) Painter, G.R.; Perryman, D.; Bluemling, G.R.WO2019173602, 2019.

6. a) Vasudevan, N.; Ahlqvist, G.P.; McGeough, C.P.; Paymode, D.J.; Cardoso, F.S.P.; Lucas, T.; Dietz, J.-P.; Opatz, T.; Jamison, T.F.; Gupton, F.B.; Snead, D.R. Chem. Commun., 2020, 56, 13363-13364. b) Gopalsamuthiram, V.; Williams, C.; Noble, J.; Jamison, T.F.; Gupton, B.F.; Snead, D.R. Synlett, 2020, 31, A-C. c) Steiner, A.; Znidar, D.; Ötvös, S.B.; Snead, D.R.; Dallinger, D.; Kappe, C.O. Eur. J. Org. Chem., 2020, 6736-6739 d) Ahlqvist, G.P.; McGeough, C.P.; Senanayake, C.; Armstrong, J.D.; Yadaw, A.; Roy, S.; Ahmed, S.; Snead, D.R.; Jamison, T.F. ACS Omega. ASAP.

7. Benkovics, T.; McIntosh, J.; Silverman, S.; Kong, J.; Maligres, P.; Itoh, T.; Yang, H.; Huffman, M.; Verma, D.; Pan, W.; Ho, H.I.; Vroom, J.; Knight, A.; Hurtak, J.; Morris, W.; Strotman, N.; Murphy, G.; Maloney, K.; Fier, P. ChemRxiv, 2020, posted on 12/22/2020. (DOI: 10.26434/chemrxiv.13472373)

Yang, C.H.; Lin, Y.C. J.Chin.Chem.Soc.1987, 34, 19-24

Paymode, D.J.; Vasudevan, N.; Ahmed, S.; Kadam, A.L.; Cardoso, F.S.P.; Burns, J.; Cook, D.W.; Stringham, R.W.; Snead, D. ChemRxiv, 2021, posted on 11/01/2021 (DOI:

10.26434/chemrxiv.13550537.v1)

\section{Corresponding author}


*Vijayagopal Gopalsamuthiram, gopalsamuv@vcu.edu 